Ann. Zootech., 1983, 32 (1), 43-52

\title{
Comparaison d'un lactoduc en ligne haute et en ligne basse. Incidence sur la traite des brebis de races Lacaune et Manech
}

\author{
J. LE DU
}

avec la collaboration technique de F.A. de la Chevalerie, J.F. Combaud et P. Lambion

I.N.R.A., Laboratoire de Recherches sur la Traite, 65, rue de Saint-Brieuc, F 35042 Rennes

\section{Résumé}

L'incidence de la hauteur du lactoduc sur les caractéristiques de traite des brebis est étudiée dans diverses conditions expérimentales : sur races Lacaune et Manech, aux niveaux de vide de 37 et de $44 \mathrm{kPa}$, avec des tuyaux de pulsation longs $(2,2 \mathrm{~m})$ et courts $(1,1 \mathrm{~m})$, avec et sans admission d'air à la griffe.

Deux expériences sont organisées selon des plans en carré latin $4 \times 4$ utilisant chacun 4 lots de 12 brebis pendant 4 périodes de 14 jours :

- Quelles que soient nos conditions expérimentales, la hauteur du lactoduc n'a pas d'incidence sur la production de lait, de matières grasses, la fréquence des chutes de gobelets et la numération cellulaire du lait (tabl. 1 et 2). De nombreuses allégations concernant les "avantages» de la ligne basse ne semblent pas justifiées lorsqu'on trait avec le matériel commercialisé actuellement (manchons moulés monoblocs, réserve de vide adéquate).

- Le niveau de vide affecte seulement la numération cellulaire du lait, plus élevée $(\mathrm{p}<0,01)$ à $44 \mathrm{kPa}$ qu'à $37 \mathrm{kPa}$. On note en particulier que la fréquence des chutes de gobelets est identique dans les deux cas.

- La race de brebis a une incidence marquée sur les chutes de gobelets. Celles-ci sont 4 fois plus fréquentes en race Lacaune qu'en race Manech (tabl. 3). Elles semblent dues ì la consistance de la mamelle plus ferme chez la brebis Lacaune que chez la brebis Manech. Il en résulte qu'un matériel qui convient pour les brebis Manech peut être inadapté pour traire des brebis Lacaune réputées plus difficiles à traire.

\section{Introduction}

Dans les salles de traite, le lactoduc peut être disposé sous le quai (ligne basse) ou au-dessus du niveau de celui-ci (ligne haute). Chez la brebis, il existe peu de données objectives concernant l'incidence de la hauteur d'évacuation du lait sur les caractéristiques de traite. 
- En race Lacaune, avec une griffe et un manchon de conception ancienne (1), Bosc aurait observé vers 1968, que la ligne basse diminue les chutes de gobelets et le volume de repasse manuelle. Il conclut que «la traite sans repasse ne peut être conseillée sur race Lacaune ou une autre race difficile à traire, sans une canalisation basse 》(Bosc, 1974).

- En race Sarde, avec un faisceau-trayeur moderne (2), CASu (1981) nobserve aucune différence importante entre la ligne basse et la ligne haute (production laitière, vidange du pis et chutes de gobelets).

Durant la traite, le contact entre la machine et l'animal se fait seulement au niveau du manchon trayeur. Il faut donc admettre que la hauteur du lactoduc ne peut avoir une incidence sur la traite qu’à condition d’influencer le mouvement du manchon ou le vide existant sous le trayon.

On sait que l'accroissement de la hauteur du lactoduc a pour effet global daugmenter les pertes de charges hydrauliques le long du faisceau trayeur. Nos études antérieures (LE Du, 1977, 1980) ont montré que le fonctionnement du manchon trayeur est affecté de la façon suivante :

- pour un réglage donné du régulateur, le vide moyen sous le trayon est moins élevé en ligne haute qu'en ligne basse;

- pour un pulsateur donné, la courbe de pulsation est plus « amortie» en ligne haute du fait de la plus grande longueur du tuyau de pulsation. Ceci se traduit par un accroissement des phases « $a$ » et « $c$ » de la courbe de pulsation définies selon la norme NF U 36000 ('s).

En conséquence, pour tenter d'expliquer les différences éventuelles, entre ligne haute et ligne basse, nous avons effectué la comparaison en étudiant simultanément l'effet du niveau de vide et de la longueur du tuyau de pulsation sur les conditions de traite des brebis de races Lacaune et Manech.

\section{Matériel et méthodes}

\section{A. Plan expérimental}

Deux expériences sont effectuées selon un même plan organisé en carré latin $4 \times 4: 4$ périodes de 14 jours, 4 traitements et 4 lots de 12 animaux chacun.

\section{Expérience $n^{\circ} 1$}

La comparaison est effectuée :

- sans admission d'air à la griffe ;

- à deux niveaux de vide différents : 37 et $44 \mathrm{kPa}{ }^{(4)}$.

(1) Griffe en bronze Alfa-Laval sans volume et sans entrée d'air. Manchon semi-extrudé Alfa-Laval avec trou freineur.

(2) Griffe en plastique Alfa-Laval $n^{\circ} 9610361$ de $48 \mathrm{ml}$, avec entrée d'air. Manchon monobloc Alfa-Laval sans trou freineur.

(3) Norme Française U 36000 d'octobre 1975 : Installations de traite mécanique. Vocabulaire. AFNOR, Tour Europe Cedex 7, 92080 Paris.

(4) 100 kilo Pascals $=75$ centimètres de mercure. 
Les 4 traitements sont :
A. Ligne haute; vide de $37 \mathrm{kPa}$;
B. Ligne haute; vide de $44 \mathrm{kPa}$;
C. Ligne basse; vide de $37 \mathrm{kPa}$;
D. Ligne basse; vide de $44 \mathrm{kPa}$.

Chacun des 4 lots de 12 animaux est composé de 6 brebis Lacaune en $1^{\text {re }}$ lactation et de 6 breb:s Manech en $3^{\circ}, 4^{\circ}$ et $5^{\circ}$ lactation.

\section{Expérience $n^{\circ} 2$}

La comparaison est effectuée :

- avec admission d'air à la griffe (7 1/min par griffe) ;

- avec deux longueurs différentes de tuyau de pulsation.

Les 4 traitements sont :
A. Ligne haute; tuyau de pulsation de $2,2 \mathrm{~m}$;
B. Ligne haute; tuyau de pulsation de $1,1 \mathrm{~m}$;
C. Ligne basse; tuyau de pulsation de $2,2 \mathrm{~m}$;
D. Ligne basse; tuyau de pulsation de $1,1 \mathrm{~m}$.

Chacun des 4 lots est composé de 12 brebis Lacaune (6 en $1^{\text {re }}$ lactation et 6 en 2" lactation).

\section{B. Matériel}

La salle de traite de type «Casse», $2 \times 12$ stalles, est équipée $2 \times 6$ faisceauxtrayeurs. D’un côté de la fosse, 6 faisceaux sont disposés en ligne haute (lactoduc $38 \times 40 \mathrm{~mm}$, à $2 \mathrm{~m}$ au-dessus du quai). De l'autre côté, 6 faisceaux sont disposés en ligne basse (lactoduc $38 \times 40 \mathrm{~mm}$, à $30 \mathrm{~cm}$ au-dessous du quai).

Lcs principales caractéristiques du matériel de traite sont les suivantes :

- pompe à vide. Capacité de $1070 \mathrm{l} / \mathrm{min}$ au vide de $44 \mathrm{kPa}$ correspondant à une réserve réelle supérieure à $750 \mathrm{l} / \mathrm{min}$ quel que soit le traitement ;

- manchons trayeurs prototypes de marque Alfa-Laval, moulés en caoutchouc noir et de forme similaire à celle du modèle n" 95953901 . Gobelets en acier inoxydable n" 96015380 ;

- griffe Fullwood constituée d'un raccord en " $Y$ », sans entrée d'air (Exp. n" 1), griffe Alfa-Laval en matière plastique avec entrée d'air de $7 \mathrm{l} / \mathrm{min}$ (Exp. $\mathrm{n}^{\prime \prime 2}$ ) ;

- tuyau à lait de $2,2 \mathrm{~m}$ en ligne haute; $1,1 \mathrm{~m}$ en ligne basse, diamètre $14 \times 7 \mathrm{~mm}$;

- tuyau de pulsation :

- exp. $\mathrm{n}^{\circ} 1: 2,2 \mathrm{~m}$ en ligne haute, $1,1 \mathrm{~m}$ en ligne basse,

- exp. $\mathrm{n}^{\circ} 2$ : 2,2 m pour les traitements $A$ et $C ; 1,1 \mathrm{~m}$ pour les traitements $\mathrm{B}$ et $\mathrm{D}$; 

de :

— pulsation : vitesse de $120 \mathrm{p} / \mathrm{min}$; rapport de $55 \mathrm{p} .100$ phases a, b, c et d

- 20,$0 ; 35,0 ; 14,2$ et 30,8 p. 100 avec tuyau de $2,2 \mathrm{~m}$,

- 18,$3 ; 36,7 ; 13,2$ et 31,8 p. 100 avec tuyau de $1,1 \mathrm{~m}$.

\section{Technique de traite}

Les gobelets sont posés sans préparation du pis. Lorsque le lait cesse de couler, un égouttage machine est pratiqué. Puis, les gobelets sont déposés et un trayeur effectue un égouttage manuel (ou repasse). La traite a lieu à 7 heures et à 16 heures.

\section{Critères mesurés}

Le contrôle des productions et des chutes de gobelets est effectué matin et soir, 3 jours par semaine aux jours $5,6,7$ et $12,13,14$ de chaque période :

- le volume de lait machine recueilli dans la chambre de réception est pesé pour chaque lot de 12 brebis (exp. 1 et 2);

- le volume d'égouttage manuel est mesuré par pesée séparément pour chaque demi-lot de 6 Lacaune et de 6 Manech (exp. 1), collectivement pour chaque lot de 12 Lacaune (exp. 2);

- le volume de lait total est la somme du lait machine et du lait d'égouttage manuel obtenu pour chaque lot de 12 brebis (exp. 1 et 2) ;

- le taux de matière grasse du lait d'égouttage manuel est mesuré collectivement pour chaque demi-lot de 6 Lacaune et de 6 Manech (exp. 1); pour chaque lot de 12 Lacaune (exp. 2);

- le taux de matières grasses du lait total est mesuré de façon à déduire la quantité totale de matières grasses pour chaque lot de 12 brebis (exp. 1 et 2);

- la fréquence des chutes de faisceaux-trayeurs est exprimée en nombre de chutes pour 100 traites. La traite d'une brebis est notée 0 si elle s'effectue sans chute et 1 lorsqu'il se produit une ou plusieurs chutes quelle que soit la cause.

Des numérations cellulaires sont effectuées au compteur Coulter matin et soir le

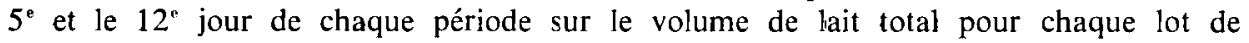
12 brebis.

\section{Résultats et discussion}

L'analyse des résultats montre que dans nos conditions expérimentales :

A. La production totale de lait et de matières grasses ainsi que le volume d'égouttage manuel ne sont pas significativement affectés par la hauteur du lactoduc, le niveau de vide ou la longueur du tuyau de pulsation (tabl. 1 et 2). En outre, ces facteurs ne semblent pas avoir d'incidence sur la vidange de la mamelle évaluée par la mesure du taux de matières grasses du lait d'égouttage.

Le principal effet physique de la ligne basse est d'accroître le vide moyen sous le trayon (LE DU, 1977). Les rares travaux existants montrent que dans la limite 
des valeurs que nous avons étudiées, le niveau de vide est sans effet sur la production et l'égouttage (Mikus, 1974; SAGI, Mor.ag \& Schmekel, 1974). Ce contexte peut expliquer la similitude des résultats obtenus en ligne haute et en ligne basse. Finalement, nous confirmons que le «freinage » du lait en ligne haute n'a pas d'incidence marquée sur la production de lait, de matières grasses et sur le volume d'égouttage (LE Du \& Bondiguel, 1978 ; LE Du et al., 1978).

\section{TABleau 1}

Effets de la hauteur du lactoduc et du niveau de vide sur les critères caractérisant la traite (exp. 1).

Effects of height of the milkline and vacuum level on milking characteristics (exp. 1).

\begin{tabular}{|c|c|c|c|c|c|}
\hline & \multirow{2}{*}{\multicolumn{2}{|c|}{$\begin{array}{c}\text { Ligne haute } \\
\text { High line } \\
\text { Vide } \\
\text { Vacuum }\end{array}$}} & \multirow{2}{*}{\multicolumn{2}{|c|}{$\begin{array}{c}\text { Ligne basse } \\
\text { Low line } \\
\text { Vide } \\
\text { Vacuum }\end{array}$}} & \multirow{3}{*}{ F } \\
\hline & & & & & \\
\hline & $37 \mathrm{kPa}$ & $44 \mathrm{kPa}$ & $37 \mathrm{kPa}$ & $44 \mathrm{kPa}$ & \\
\hline $\begin{array}{l}\text { Lait total }(\mathrm{g} / \mathrm{brebis} / \text { jour }) \\
\text { Total milk }(g / \text { ewe/day })\end{array}$ & 717 & 695 & 703 & 717 & $0,55 \mathrm{NS}$ \\
\hline $\begin{array}{l}\text { Lait machine (g/brebis/jour) } \ldots . . \\
\text { Machine milk (g/ewe/day) }\end{array}$ & 644 & 625 & 638 & 656 & $0,91 \mathrm{NS}$ \\
\hline $\begin{array}{l}\text { Egouttage manuel (g/brebis/jour) .. } \\
\text { Hand stripping (g/ewe/day) }\end{array}$ & 72,9 & 69,9 & 65,5 & 61,0 & $2,68 \mathrm{NS}$ \\
\hline $\begin{array}{l}\text { Matières grasses totales } \ldots \ldots \ldots \ldots \\
\text { (g/brebis/jour) } \\
\text { Total fat }(g / \text { ewe/day })\end{array}$ & 44,3 & 42,4 & 44,3 & 44,9 & $2,18 \mathrm{NS}$ \\
\hline $\begin{array}{l}\text { MG, lait d'égouttage (g/litre) } \\
\text { Stripping fat }(\mathrm{g} / \text { litre })\end{array}$ & 99,4 & 99,4 & 99,6 & 99,6 & $0,00 \mathrm{NS}$ \\
\hline $\begin{array}{l}\text { Cellules totales }(1000 \mathrm{ml}) \ldots \ldots \ldots \\
\text { Cell counts }(1000 / \mathrm{ml})\end{array}$ & $280_{\mathrm{a}}$ & $462_{b}$ & $309_{a}$ & $568_{\mathrm{b}}$ & $27,18 * *$ \\
\hline $\begin{array}{l}\text { Chutes des gobelets (pour } 100 \text { traites) } \\
\text { Cluster falls (for } 100 \text { milkings) }\end{array}$ & 11,4 & 8,5 & 7,0 & 7,7 & $2,27 \mathrm{NS}$ \\
\hline
\end{tabular}

NS : non significatif au seuil $0,05(\mathrm{~F}<4,76)$ - non significant at the 0.05 level $(F<4.76)$.

(**) : significatif au seuil $0,01 \quad(\mathrm{~F}<9,78)$ - significant at the 0.01 level $(F<9.78)$.

Cellules totales : les valeurs repérées par la même lettre ne diffèrent pas significativement à $\mathrm{p}=0,01$ (Test de DUNCAN) - Cell counts: the values followed by the same letter are not significantly different at $p=0.01$ (DUNCAN's method). 


\section{TABLEAU 2}

Effets de la hauteur du lactoduc et de la longueur du tuyau à pulsation sur les critères caractérisant la traite (exp. 2).

Effects of height of the milkline and length of the pulsation tube on milking characteristics (exp. 2).

\begin{tabular}{|c|c|c|c|c|c|}
\hline & \multirow{2}{*}{\multicolumn{2}{|c|}{$\begin{array}{l}\text { Ligne haute } \\
\text { High line } \\
\text { Tuyau de pulsation } \\
\text { Pulsation tube }\end{array}$}} & \multirow{2}{*}{\multicolumn{2}{|c|}{$\begin{array}{l}\text { Ligne basse } \\
\text { Low line } \\
\text { Tuyau de pulsation } \\
\text { Pulsation tube }\end{array}$}} & \multirow{3}{*}{$\mathrm{F}$} \\
\hline & & & & & \\
\hline & $2,2 \mathrm{~m}$ & $1,1 \mathrm{~m}$ & $2,2 \mathrm{~m}$ & $1,1 \mathrm{~m}$ & \\
\hline $\begin{array}{l}\text { Lait total }(\mathrm{g} / \mathrm{brebis} / \text { jour }) \\
\text { Total milk }(\mathrm{g} / \text { ewe/day })\end{array}$ & 1034 & 1013 & 1038 & 1042 & $1,76 \mathrm{NS}$ \\
\hline $\begin{array}{l}\text { Lait machine }(\mathrm{g} / \mathrm{brebis} / \text { jour }) \ldots \ldots \\
\text { Machine milk }(\mathrm{g} / \text { ewe/day })\end{array}$ & 994 & 970 & 996 & 1000 & $1,73 \mathrm{NS}$ \\
\hline $\begin{array}{l}\text { Egouttage manuel ( } \mathrm{g} / \text { brebis/jour) } \\
\text { Hand stripping }(\mathrm{g} / \mathrm{ewe} / \text { day })\end{array}$ & 40,0 & 43,1 & 42,6 & 42,4 & $0,47 \mathrm{NS}$ \\
\hline $\begin{array}{l}\text { Matières grasses totales } \ldots \ldots \ldots \ldots \\
(\mathrm{g} / \text { brebis/jour) } \\
\text { Total fat }(\mathrm{g} / \text { ewe/day })\end{array}$ & 77,4 & 77,4 & 78,8 & 77,9 & $0,74 \mathrm{NS}$ \\
\hline $\begin{array}{l}\text { MG, lait d'égouttage }(\mathrm{g} / \text { litre }) \ldots \ldots \\
\text { Siripping fat }(\mathrm{g} / \text { litre })\end{array}$ & 141,6 & 141,9 & 143,8 & 138,1 & $1,55 \mathrm{NS}$ \\
\hline $\begin{array}{l}\text { Cellules totales }(1000 / \mathrm{ml}) \ldots \ldots \\
\text { Cell counts }(1000 / \mathrm{ml})\end{array}$ & 670 & 862 & 836 & 675 & $0,20 \mathrm{NS}$ \\
\hline $\begin{array}{l}\text { Chutes des gobelets (pour } 100 \text { traites) } \\
\text { Cluster falls (for } 100 \text { milkings) }\end{array}$ & 22 & 17 & 22 & 19 & $0,38 \mathrm{NS}$ \\
\hline
\end{tabular}

NS : non significatif au seuil $0,05(\mathrm{~F}<4,76)$ - non significant at the 0.05 level $(F<4.76)$.

La différence de longueur entre tuyaux de pulsation semblait susceptible d'expliquer un effet éventuel de la hauteur du lactoduc. Phillips (1963) a notamment montré chez la vache que l'égouttage est diminué de 20 p. 100 lorsque la durée de la transition massage-succion (phase a) passe de 18 à 8 p. 100 du cycle. Dans nos conditions expérimentales, avec des tuyaux de pulsation utilisés normalement en ligne haute (longueur 2,2 $\mathrm{m}$ ) et en ligne basse (longueur $1,1 \mathrm{~m}$ ), on mesure des phases a de 20,0 p. 100 et de 18,3 p. 100 . Compte tenu de nos résultats, il semble que cette différence soit trop faible pour affecter le mouvement du manchon et par conséquent les conditions d'écoulement du lait.

B. La numération cellulaire du lait n'est pas significativement affectée par la hauteur du lactoduc ou par la longueur du tuyau de pulsation (tabl. 1 et 2). Par 
contre, en ligne haute comme en ligne basse, elle est accrue significativement $(p<0,01)$ lorsque le niveau de vide passe de 37 à $44 \mathrm{kPa}$ (tabl. 1). Il est à noter que cet effet est observé durant la $1^{\text {ro }}$ expérience en trayant sans admission d'air à la griffe. La même expérience montre que le nombre de cellules a également tendance (non significatif) à être plus élevé en ligne basse qu'en ligne haute. Cet effet n'apparaît pas lorsqu'on trait avec admission d'air à la griffe (tabl. 2). Nous avons antérieurement montré que l'admission d'air a pour effet de réguler le vide moyen sous le trayon en l'accroissant en ligne haute et en le diminuant en ligne basse (LE DU, 1977 ; LE DU \& Bondiguel, 1978). L'admission d'air à la griffe aurait donc pour effet d'atténuer les différences éventuelles entre les deux systèmes de traite.

C. Les chutes de gobelets ne sont pas significativement affectées par la hauteur du lactoduc, le niveau de vide ou la longueur du tuyau de pulsation (tabl. 1 et 2). La bibliographie concerne uniquement l'effet du niveau de vide et confirme nos résultats. Avec des vides de 44 et $33 \mathrm{kPa}$, similaires à ceux que nous avons étudiés (44 et $37 \mathrm{kPa}$ ), Mikus (1974) compte en moyenne 12 p. 100 de chutes. Par contre, la fréquence de celles-ci est nettement accrue lorsque le niveau de vide devient anormalement faible $(22$ p. 100 à $27 \mathrm{kPa})$.

La fréquence des chutes est en moyenne 4 fois plus élevée avec des brebis Lacaune qu'avec les brebis Manech (tabl. 3). Ce résultat est établi en comparant des brebis Lacaune primipares et des brebis Manech multipares (exp. 1). Le facteur race apparaît toutefois plus important que le numéro de lactation car la $2^{e}$ expérience montre qu'il existe peu de différence entre les brebis Lacaune primipares (16 p. 100 de chutes) et multipares ( 24 p. 100 de chutes).

\section{TABLEAU 3}

Fréquence des chutes de faisceaux-trayeurs en races Lacaune et Manech. Effets de la hauteur du lactoduc et du niveau de vide (exp. 1).

Cluster fall frequency for Lacaune and Manech breeds Effects of height of the milkline and vacuum level (exp. 1).

\begin{tabular}{|c|c|c|c|c|c|c|}
\hline & & \multirow{2}{*}{\multicolumn{2}{|c|}{$\begin{array}{c}\text { Ligne haute } \\
\text { High line } \\
\text { Vide } \\
\text { Vacuum }\end{array}$}} & \multirow{2}{*}{\multicolumn{2}{|c|}{$\begin{array}{c}\text { Ligne basse } \\
\text { Low line } \\
\text { Vide } \\
\text { Vacuum }\end{array}$}} & \multirow{3}{*}{$\begin{array}{l}\text { Niveau } \\
\text { de signif. } \\
\text { Level of } \\
\text { signif. }\end{array}$} \\
\hline & & & & & & \\
\hline & & $37 \mathrm{kPa}$ & $44 \mathrm{kPa}$ & $37 \mathrm{kPa}$ & $44 \mathrm{kPa}$ & \\
\hline \multirow{3}{*}{$\begin{array}{l}\text { Chute des gobelets } \\
\text { (pour } 100 \text { traites) } \\
\text { Cluster falls } \\
\text { (for } 100 \text { milkings) }\end{array}$} & $\begin{array}{l}\text { Race Lacaune } \\
\text { Lacaune breed }\end{array}$ & 17,8 & 14,6 & 10,9 & 11,6 & NS \\
\hline & $\begin{array}{l}\text { Race Manech .... } \\
\text { Manech breed }\end{array}$ & 5,0 & 2,3 & 3,1 & 3,7 & NS \\
\hline & $\begin{array}{l}\text { Niveau de signif. } \\
\text { Level of signif. }\end{array}$ & $* *$ & $* *$ & $* *$ & $* *$ & \\
\hline
\end{tabular}

NS : non significatif au seuil 0,05 - non significant at the 0.05 level.

(**) : significatif au seuil 0,01 - significant at the 0.01 level. 
Les chutes semblent plus fréquentes lorsque le pis est dur au toucher : c'est le cas de la brebis Lacaune dont la citerne est plus petite et plus ferme que celle de la brebis Manech.

\section{Conclusion}

Dans nos conditions expérimentales, les caractéristiques de traite que nous avons mesurées sont globalement similaires en ligne haute et en ligne basse. La disposition du lactoduc n'a pas d'incidence sur la production laitière totale, la production de matières grasses, le volume de repasse, la fréquence des chutes de faisceaux-trayeurs et la numération cellulaire du lait. Ces conclusions sont valables au vide de 37 et de $44 \mathrm{kPa}$, avec et sans admission d'air à la griffe, pour les races de brebis Lacaune et Manech. Par ailleurs, il ne semble pas que l'on puisse justifier un éventuel «avantage » de la ligne basse par le vide plus élevé sous le trayon ou l'aspect moins «amorti»de la courbe de pulsation.

Finalement, l'absence globale de différences entre la ligne haute et la ligne basse contredit les observations de Bosc (1974), qui considère dès 1968 la ligne basse comme un perfectionnement susceptible de diminuer les chutes et le volume d'égouttage manuel. La contradiction n'est peut-être qu'apparente. A l'époque, la capacité des pompes à vide était relativement faible et tous les faisceaux trayeurs étaient conçus pour freiner l'écoulement du lait de façon à éviter les chutes simultanées de gobelets (LE Du et al., 1978). Par contre, l'installation utilisée pour nos essais a une réserve de vide importante $(750 \mathrm{l} / \mathrm{min})$ et est équipée de manchons monoblocs de conception récente. Avec un équipement similaire au nôtre, CASU (1981) considère que la hauteur du lactoduc n'a pas d'incidence sur la traite des brebis Sardes. Il semble donc que l'évolution du matériel de traite puisse expliquer la contradiction entre nos résultats et les observations faites dans la région de Roquefort vers 1968.

En pratique, on peut considérer que la disposition du lactoduc affecte surtout l'organisation du travail en fosse de traite. En ligne basse, il est obligatoire de disposer les faisceaux-trayeurs de chaque côté de la fosse (double équipement). En ligne haute, ceux-ci peuvent être disposés au centre de la fosse et transférés d'une brebis à l'autre en étant utilisés à plein temps (simple équipement). II en résulte qu'à productivité horaire donnée, une installation en ligne basse nécessite plus de faisceaux-trayeurs qu'une installation en ligne haute. Actuellement, de nombreux éleveurs considèrent que la ligne basse présente des inconvénients du point de vue du montage, du nettoyage et de l'accessibilité au pis. Une certaine préférence se dégage donc en faveur de la ligne haute. Une étude de marché (GaUTIER, 1980) portant sur 275 éleveurs de Roquefort souhaitant acquérir un système «Casse» dans les 5 ans montre que 87 p. 100 d'entre eux se prononcent en faveur de la ligne haute.

Le niveau de vide, choisi dans la gamme des valeurs couramment utilisées (37 ou $44 \mathrm{kPa}$ ) n'a pas d'incidence significative sur la production de lait, de matières grasses ou sur la fréquence des chutes de gobelets. Par contre, le vide le plus élevé conduit à une augmentation $(p<0,01)$ de la numération cellulaire du lait, indice d'une «irritation» du trayon. Compte tenu de ces observations, il semble judicieux de choisir un niveau de vide relativement faible. Celui-ci doit toutefois être suffisant pour assurer une stabilité satisfaisante du faisceau-trayeur sur le pis. 
Enfin, le facteur race apparaît avoir une grande incidence sur les chutes de faisceaux-trayeurs. Celles-ci sont 4 fois plus fréquentes avec des brebis de race Lacaune qu'avec des brebis de race Manech. La consistance plus ferme du pis de la brebis Lacaune semble causer cette différence. Il en résulte qu'un matériel de traite doit dans une certaine mesure être conçu en fonction de la race de brebis à traire. Un faisceautraveur qui donne satisfaction sur des races de type Manech, faciles à traire peut ne pas convenir au cas particulier de la brebis de race Lacaune, réputée difficile à traire.

Accepté pour publication en décembre 1982.

\section{Remerciements}

Nous exprimons notre reconnaissance au personnel de la bergerie du Rheu pour la collaboration précieuse apportée à la réalisation de ce travail.

\section{Summary}

Comparison of highline and lowline milking. Effect on milking characteristics of Lacaune and Manech ewes

The effect of milkline height was studied under several experimental conditions : on Lacaune and Manech ewes, at vacuum levels of 37 and $44 \mathrm{kPa}$, with long $(2.2 \mathrm{~m})$ and short $(1.1 \mathrm{~m})$ pulsation tubes, with and without air admission at the claw.

Two experiments were done, each of them using a $4 \times 4$ latin square, with 4 groups of 12 ewes milked during 4 periods of 14 days.

- Whatever the experimental conditions, the milkline height had no significant incidence on milk and fat production, cluster fall frequency and milk cell count (tabl. 1 and 2).

- The only effect of the vacuum level was an increase in milk cell count, significantly higher for $44 \mathrm{kPa}$ than for $37 \mathrm{kPa}$ (fig. 1). Cluster fall frequency was not affected.

- Cluster falls were mainly affected by the breed. They were 4 times more frequent with Lacaune than with Manech (tabl. 3). This effect seemed to be related to the consistency of the udder which is stiffer in Lacaune ewes. Thus, an equipment which gives good results for the Manech breed could be unsuitable for the Lacaune breed, known as being. more difficult to milk.

\section{Références bibliographiques}

Bosc M., 1974. Organisation et productivité du travail de la traite des brebis laitières. Importance du choix d'une méthode de traite. $I^{\text {er }}$ Symposium International sur la Traite Mécanique des Petits Ruminants. Ann. Zootech., numéro hors série, 231-252.

Casu S., 1981. Communication personnelle.

Gautier P., 1980. Etude de marché : salle de traite alternative de type Leducteur. Rapport de stage, I.N.A. Paris-Grignon, 47 p.

LE Du J., 1977. Machine à traire : paramètres physiques caractérisant le fonctionnement du manchon trayeur. Ann. Biol. anim. Biochim. Biophys., 17, 971-985. 
LE Du J., 1980. Effect of some components of the milking machine on irregular vacuum perturbations recorded at the teat level. Int. Workshop on Mach. Milking and Mastitis, Moorepark, Ireland, 82-90.

Le Du J., Bondiguel L., 1978. Machine à traire pour brebis : variation du vide sous le trayon avec la pulsation, l'entrée d'air à la griffe et le volume collecteur du lait. $2^{e}$ Symposium International sur la Traite Mécanique des Petits Ruminants, F.N.O.C.L., Paris, 346-362.

Le Du J., Labussière J., Petrequin P., Mrrman B., Combaud J.F., 1978. Effets de la pulsation, du mouvement du manchon et des conditions d'écoulement du lait sur la traite des brebis Préalpes du Sud. $2^{e}$ Symposium International sur la Traite Mécanique des Petits Ruminants, F.N.O.C.L., Paris, 363-384.

Mikus M., 1974. Machine à traire les brebis et les chèvres. $1^{\text {er }}$ Symposium International sur la Traite Mécanique des Petits Ruminants. Ann. Zootech., numéro hors série, 181-194.

Phillips D.S.M., 1963. The effect of pulsator ratio on milking rate. Proc. of Ruakura Farmers Conference Week, 1-15.

SAGi R., Morag M., Schmekel J., 1974. Effet des paramètres de la machine à traire sur la production laitière et le fractionnement du lait chez les brebis laitières. $1^{\text {er }}$ Symposium International sur la Traite Mécanique des Petits Ruminants. Ann. Zootech., numéro hors série, 195-200. 